\title{
Dosimetric comparison between intensity-modulated radiotherapy and RapidArc with single arc and dual arc for malignant glioma involving the parietal lobe
}

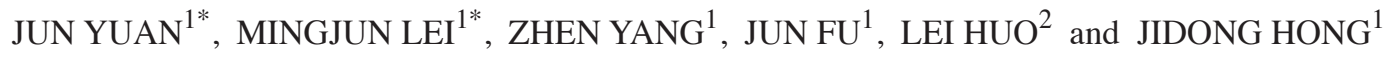 \\ ${ }^{1}$ Department of Radiation Oncology, ${ }^{2}$ The Institute of Skull Base Surgery and Neuro-Oncology at Hunan, \\ Xiangya Hospital, Central South University, Changsha, Hunan 410008, P.R. China
}

Received December 15, 2015; Accepted April 11, 2016

DOI: $10.3892 / \mathrm{mco} .2016 .872$

\begin{abstract}
The aim of the present study was to evaluate the difference in treatment plan quality, monitor units (MUs) per fraction and dosimetric parameters between IMRT (intensity-modulated radiotherapy) and RapidArc with single arc (RA1) and dual arc (RA2) for malignant glioma involving the parietal lobe. Treatment plans for IMRT and RA1 and RA2 were prepared for 10 patients with malignant gliomas involving the parietal lobe. The Wilcoxon matched-pair signed-rank test was used to compare the plan quality, monitor units and dosimetric parameters between IMRT and RA1 and RA2 through dose-volume histograms. Dnear-max (D2\%) to the left lens, right lens and left optical nerve in RA1 were less compared with those in IMRT; D2\% to the right lens and right optic nerve in RA2 were less compared with those in IMRT. D2\% to the optic chiasma in RA2 was small compared with that in RA1. The median dose (D50\%) to the right lens and right optic nerve in RA1 and RA2 was less compared with the identical parameters in IMRT, and D50\% to the brain stem in RA2 was less compared with that in RA1. The volume receiving at least $45 \mathrm{~Gy}$ (V45) or V50 in normal brain tissue (whole brain minus the planning target volume 2; B-P) in RA1 was less compared with that in IMRT. V30, V35, V40, V45, or V50 in B-P in RA2 was less compared with that in IMRT. The MUs per fraction in RA1 and RA2 were significantly less compared with those in IMRT. All differences with a P-value $<0.05$ were considered to be significantly different. In conclusion, RA1 and RA2 markedly reduced the MUs per fraction, and spared partial organs at risk and B-P compared with IMRT.
\end{abstract}

Correspondence to: Dr Jidong Hong, Department of Radiation Oncology, Xiangya Hospital, Central South University, 87 Xiangya Road, Changsha, Hunan 410008, P.R. China

E-mail: hongjidong1966@126.com

*Contributed equally

Key words: malignant glioma, dosimetry, intensity-modulated arc radiotherapy, intensity-modulated radiotherapy

\section{Introduction}

Treatment for malignant gliomas typically requires a combined approach that includes surgery, radiotherapy and chemotherapy. Radiotherapy is an important adjuvant treatment for malignant gliomas. Intensity-modulated radiotherapy (IMRT) has been demonstrated to be superior to three-dimensional conformal radiotherapy (3D-CRT) in patients with malignant gliomas. MacDonald et al (1) compared the dosimetric distribution of non-coplanar IMRT in malignant gliomas with that of 3D-CRT, and identified that non-coplanar IMRT improved the target coverage and reduced the radiation dose to the brain, brainstem and optic chiasm. Lorentini et al (2) performed a dosimetric comparison between IMRT and 3D-CRT in glioblastoma. IMRT appears to be a superior radiation technique compared with 3D-CRT when multiple overlaps exist between the planning target volume (PTV) and organs at risk (OARs). IMRT allows for improved target coverage while maintaining equivalent OARs, sparing and reducing normal brain irradiation. Intensity-modulated arc radiotherapy (IMAT) represents the latest evolution of cancer treatment technology, setting novel benchmarks for speed, precision and patient comfort. IMAT, which at Varian Medical Systems, Inc. (Palo Alto, CA, USA) is termed RapidArc, is similar to Elekta's (Stockholm, Sweden) Elekta Synergy ${ }^{\circledR}$ volumetric-modulated arc therapy (VMAT) and Philips' (Amsterdam, The Netherlands) Pinnacle ${ }^{3}$ SmartArc treatment planning solution. RapidArc uses a unique algorithm that provides unprecedented treatment delivery control. As a result, treatment plans that excel in covering target goals, while sparing critical structures, can be developed with performance speeds faster than ever before. Clinicians are able to develop treatments that take one-half to one-eighth the time of conventional IMRT treatments: Only $2 \mathrm{~min}$ in a number of cases. IMAT treatment may also result in less radiation leakage and scatter, so that peripheral tissues receive a lower overall dose. IMAT was used to evaluate the effect on dosage distributions in OARs and normal brain tissue compared with IMRT and 3D-CRT in high-grade gliomas, which were predominantly located in the frontal and temporal lobes of the cerebral hemisphere $(3,4)$. In order to compare the dosimetric parameters of IMRT with those of RapidArc with single arc (RA1) and dual arc (RA2) 
in malignant gliomas involving the parietal lobe, in the present study IMRT, RA1 and RA2 treatment plans were developed for each of 10 patients with malignant glioma.

\section{Materials and methods}

Patient selection and delineation of the PTV and OARs. A total of 10 patients (five men and five women) with malignant glioma involving the parietal lobe were enrolled in the present study. The study was approved by the Medical Ethics Committee of Xiangya Hospital of Central South University and all participants gave written content. All the participants had been surgically treated, and their condition was confirmed by pathological diagnosis. Their ages ranged from 16 to 59 years (mean age, 45.8 years). According to the World Health Organization (WHO) 2007 classification of tumors of the central nervous system (CNS), there were five cases of grade III and five cases of grade IV (5). Temozolomide was used in all patients as adjuvant chemotherapy to surgery and radiotherapy, referring to Stupp's method (6). Patients with malignant glioma received concomitant chemotherapy consisting of daily temozolomide $\left(75 \mathrm{mg} / \mathrm{m}^{2} /\right.$ day) with IMRT or RapidArc and adjuvant chemotherapy consisting of up to six cycles of maintenance temozolomide $\left(150-200 \mathrm{mg} / \mathrm{m}^{2} /\right.$ day on days $1-5$ repeated every 28 days). The clinical data of the 10 patients with malignant glioma are shown in Table I.

Patients were scanned with simulated computed tomography (CT) using a Somatom Definition AS CT scanner (Siemens AG, Munich, Germany) at a 3-mm slice thickness, with T1-weighted magnetic resonance imaging (MRI) using a Magnetom Sonata 1.5T MRI scanner (Siemens AG), with contrast being performed in the meantime and registered with CT. The gross tumor volume tumor bed (GTVtb) was contoured as the residual tumor and postoperative tumor bed according to the operative record, preoperative MRI and postoperative MRI within 3 days following the surgery; the GTVtb with $0.5 \mathrm{~cm}$ margins was identified as the planning (P)GTVtb. The clinical target volume 1 (CTV1) was outlined as the GTVtb with 1.5-2.0 cm margins, and the CTV2 was delineated as the GTVtb with 2-2.5 cm margins; CTV1 and CTV2 were based on the pathological grades of gliomas and limitation of dose to OARs. Dose limitation to OARs was undertaken with reference to the Radiation Therapy Oncology Group 0825 protocol. (7). CTV1 and CTV2 were expanded with $0.5 \mathrm{~cm}$ margins, resulting in the PTV1 and PTV2, respectively. OARs included the brainstem, bilateral lenses, bilateral optic nerve, bilateral hippocampus, optic chiasm, pituitary gland and normal brain tissue [which meant the whole brain minus PTV2, or B-P)]. The brainstem, bilateral lens and optic chiasm with $0.3 \mathrm{~cm}$ margins were created as the brainstem planning risk volume (PRV), the bilateral lens PRV and the optical chiasm PRV, respectively.

Prescribed doses, plan objective and OAR constraints. PTVs were divided into various subPTVs, including the PGTVtb, PTV1 and PTV2, as described above, which delivered various prescribed doses of radiation. PGTVtb received $64.2 \mathrm{~Gy}$ in 30 fractions (2.14 Gy per fraction), whereas PTV1 received 60 Gy in 30 fractions (2 Gy per fraction); and PTV2 received $54 \mathrm{~Gy}$ in 30 fractions (1.8 Gy per fraction) using the simultaneous integrated boost technique. Measured as a percentage, $95 \%$ of the PTV received $95 \%$ of the prescribed dose; the volume of PTV that received $\geq 110 \%$ of the prescribed dose was $<20 \%$; the volume of PTV that received $\leq 93 \%$ of the prescribed dose was $<3 \%$; and areas outside of the PTV were not allowed to receive $>110 \%$ of the prescribed dose. The maximum dose (Dmax) to the brainstem was limited to $54 \mathrm{~Gy}$; Dmax to the lens was limited to $9 \mathrm{~Gy}$; and Dmax to the optical nerve, optical chiasm and pituitary gland were limited to $50 \mathrm{~Gy}$.

Planning techniques. The IMRT, RA1 and RA2 treatment plans were designed by using the identical CT data fused with regular MRI T1-weighted images contrasted for every patient on the Varian Eclipse ${ }^{\mathrm{TM}}$ treatment planning system (version 8.6.05; Varian Medical Systems, Inc.) with $6 \mathrm{MV}$ photon beams from a Varian Trilogy, respectively. The prescription and planning objectives used for the three treatment plans were identical.

IMRT was computed with a fixed gantry, with the couch angle set to $0^{\circ}$ and the collimator set at $10^{\circ}$; the type of multileaf collimator (MLC) was the Varian Millennium 120 leaf MLC (Varian Medical Systems, Inc.). MLC leaf sequences were generated using the dynamic sliding window IMRT delivery $(8,9)$. Plans were individually optimized by using seven co-planar fields selecting for the best geometry for each patient. A fixed dose rate (DR) of 600 monitor units (MUs)/min was selected for IMRT.

RA1 used a single-arc rotation intensity-modulated technology, consisting of a single $360^{\circ}$ rotation (clockwise) with the couch angle set to $0^{\circ}$ and the collimator set to $10^{\circ}$. The arc starts with a gantry angle of $181^{\circ}$, and stops at a gantry angle of $179^{\circ}$. RA2 used a dual-arc rotation intensity-modulated technique, consisting of two co-planar arcs of $360^{\circ}$ optimized simultaneously to be delivered with opposite rotation (clockwise and counter-clockwise). For the RA2 plans, the couch was set to $0^{\circ}$ for the two arcs, whereas the collimator rotation was set to the identical angle as in the RA1 plans for the first arc and to $325^{\circ}$ for the second arc. The first arc (clockwise) started with a gantry angle of $181^{\circ}$, and stopped at a gantry angle of $179^{\circ}$. The second arc (counter-clockwise) started with a gantry angle of $179^{\circ}$ and stopped at a gantry angle of $181^{\circ}$. Plans for RA1 and RA2 were optimized by selecting a maximum DR of $600 \mathrm{MU} / \mathrm{min}$.

The anisotropic analytical algorithm (AAA) was used for IMRT, RA1 and RA2 (10-12). The dose calculation grid was set to $0.125 \mathrm{~cm}(13)$.

Plan quality evaluation, dose distribution and parameter comparison. Dose-volume histograms (DVHs) of IMRT, RA1 and RA2 were generated with use of the Eclipse ${ }^{\mathrm{TM}}$ Treatment system (Varian Medical Systems, Inc.). Comparisons of dosimetric parameters and plan quality were performed among IMRT, RA1 and RA2, and the conformal index (CI) was calculated according to the method described by van't Riet et al (14): $\mathrm{CI}=\mathrm{TV}_{\mathrm{RI}}{ }^{2} / \mathrm{TVxV}_{\mathrm{RI}}$, where $\mathrm{TV}_{\mathrm{RI}}$ is the target volume covered by the reference isodose, $\mathrm{TV}$ is the target volume and $\mathrm{V}_{\mathrm{RI}}$ is the volume of the reference isodose; higher values of CI represented an improved PTV conformality. The homogeneity index (HI) refers to the formula described by $\mathrm{Wu}$ et al (15): $\mathrm{HI}=\left(\mathrm{D}_{2 \%}-\mathrm{D}_{98 \%}\right) / \mathrm{D}_{\mathrm{p}}$, where $\mathrm{D}_{\mathrm{p}}$ is the prescription 
Table I. Clinical characteristics of the 10 patients with malignant glioma.

\begin{tabular}{|c|c|c|c|c|c|c|c|}
\hline No. & Gender & $\begin{array}{c}\text { Age } \\
\text { (years) }\end{array}$ & Side & Location & $\begin{array}{l}\text { Size } \\
\left(\mathrm{cm}^{2}\right)\end{array}$ & $\begin{array}{c}\text { Extent } \\
\text { of surgery }\end{array}$ & $\begin{array}{c}\text { Pathological } \\
\text { grade }\end{array}$ \\
\hline 1 & $\mathrm{~F}$ & 44 & Right & Parietooccipital lobe & $4.1 \times 5.1$ & GTR & III \\
\hline 2 & M & 58 & Right & Parietooccipital lobe & $2.0 \times 2.0$ & PR & IV \\
\hline 3 & $\mathrm{~F}$ & 26 & Right & Parietofrontal lobe & $3.0 \times 4.0$ & GTR & IV \\
\hline 4 & M & 45 & Left & Parietofrontal lobe & $4.5 \times 4.0$ & PR & III \\
\hline 5 & M & 40 & Right & Parietotemporal lobe & $8.0 \times 6.5$ & GTR & III \\
\hline 6 & M & 16 & Left & Parietal lobe & $2.6 \times 2.1$ & PR & III \\
\hline 7 & $\mathrm{~F}$ & 59 & Left & Parietotemporal and frontal lobe & $5.0 \times 7.0$ & PR & IV \\
\hline 8 & $\mathrm{~F}$ & 56 & Left & Parietotemporal, and occipital lobe & $4.0 \times 4.5$ & GTR & IV \\
\hline 9 & $\mathrm{~F}$ & 58 & Right & Parieto frontal lobe & $4.0 \times 5.0$ & PR & III \\
\hline 10 & M & 56 & Left & Parietotemporal lobe & $5.0 \times 4.0$ & PR & IV \\
\hline
\end{tabular}

No., patient number; M, male; F, female; GRT, gross tumor resection; PR, partial resection.

dose, Dnear-max $\left(\mathrm{D}_{2 \%}\right)$ is the dose/2\% volume of PTV received, and Dnear-min $\left(\mathrm{D}_{98 \%}\right)$ is the dose/98\% volume of PTV received; lower values of HI represented an improved PTV homogeneity. median dose (D50\%) was the dose $/ 50 \%$ volume of PTV. D2\%, D50\%, V5, V10, V15, V20, V25, V30, V35, V40, V45 and V50 of B-P were compared among IMRT, RA1 and RA2; Vn refers to the volume of the B-P receiving at least $\mathrm{nGy}$.

Statistical analysis. SPSS 13.0 software (SPSS, Inc., Chicago, IL, USA) was used to perform the statistical analysis. Statistical tests of differences between dosimetric parameters of IMRT, RA1 and RA2 were evaluated using a two-sided Wilcoxon matched-pair signed-rank test (each pair in the test consisting of the patient-specific dosimetric parameters for IMRT, RA1 and RA2). $\mathrm{P}<0.05$ was considered to indicate a statistically significant difference.

\section{Results}

In the present study, with respect to the D2\% to OARs, the D2\% values to the left lens, right lens and left optic nerve in RA1 were significantly less compared with those in IMRT $(\mathrm{P}<0.05)$, respectively (Table II). D2\% to the right lens and right optic nerve in RA2 were significantly less compared with those in IMRT $(\mathrm{P}<0.05)$. D2\% to the optic chiasma in RA2 was significantly less compared with that in RA1 $(\mathrm{P}<0.05)$. With respect to the $\mathrm{D} 50 \%$ to OARs, the D50\% to the right lens and right optic nerve in RA1 and RA2 were significantly less compared with those in IMRT $(\mathrm{P}<0.05)$. D50\% to the brainstem in RA2 was significantly less compared with that in RA1 $(\mathrm{P}<0.05)$; in addition, V45 and V50 of B-P from RA1 were less compared with those from IMRT, with statistically significant differences $(\mathrm{P}<0.05)$. V30-V50 of B-P in RA2 were significantly less compared with those in IMRT $(\mathrm{P}<0.05)$, respectively. Without prospectively optimizing to spare the hippocampus, D2\% and D50\% to the right and left hippocampi did not yield any significant differences among IMRT, RA1 and RA2, which indicated that the hippocampus is not affected by different radiotherapy techniques that feature no effort to spare it (Table II).
The dose distributions of one representative patient generated by IMRT, RA1 and RA2 are shown in Fig. 1. D2\% and $\mathrm{D} 50 \%$ of OARs, with significant differences $(\mathrm{P}<0.05)$ are shown in Fig. 2. The mean DVHs for the OARs of all the patients treated with different radiotherapy techniques are shown in Fig. 3. In terms of CI, HI of subPTV and MUs per fraction, all $\mathrm{CI}$ and $\mathrm{HI}$ values of subPTV in RA1 were less compared with those in IMRT $(\mathrm{P}<0.05)$; by contrast, all CI and HI values of subPTV in RA2 were similar to those in IMRT, and they were not significantly different $(\mathrm{P}>0.05)$ (Table III). Therefore, this suggests that, although RA did not improve the coverage and homogeneity of the target volume with sparing OARs, RA markedly reduced the MUs per fraction compared with IMRT $(\mathrm{P}<0.05)$, and no significant differences in MUs per fraction were identified between RA1 and RA2. RA1 and RA2 significantly decreased the treatment times compared with those of IMRT; the treatment time of RA1 was lower compared with that of RA2, with a significant difference noted $(\mathrm{P}<0.05)$. Data for the parameters CI, HI of PTV, MUs per fraction and treatment times in IMRT, RA1, and RA2 are shown in Table III.

\section{Discussion}

IMAT (RapidArc; Varian Medical Systems, Inc.) has been increasingly used for numerous types of tumors from different anatomical sites, including those in the CNS. Shaffer et al (3) compared the treatment plans in 10 cases with frontal and temporal high-grade gliomas between VMAT with single arc and IMRT. PTV coverage, conformality and homogeneity were shown to be equivalent in VMAT and IMRT. VMAT significantly reduced the maximum and mean retinal, lens and contralateral optic nerve doses compared with IMRT $(\mathrm{P}<0.05)$, whereas the brainstem, chiasm and ipsilateral optic nerve doses were similar. VMAT significantly reduced the mean MUs and treatment time compared with IMRT. The results of the present study are similar to those of Shaffer et al (3) on the whole; however, the CI and HI in RA1 were inferior to those in IMRT. One explanation may be that the different location of the gliomas led to different results. Wagner et al (16) analyzed 11 cases of 
Table II. Dosimetric parameters of IMRT, RA1 and RA2.

\begin{tabular}{|c|c|c|c|c|c|c|}
\hline Parameter & $\begin{array}{c}\text { IMRT, } \\
\text { mean } \pm \text { SD }\end{array}$ & $\begin{array}{l}\text { P for IMRT } \\
\text { vs. RA1 }\end{array}$ & $\begin{array}{c}\mathrm{RA} 1, \\
\text { mean } \pm \mathrm{SD}\end{array}$ & $\begin{array}{l}\text { P for IMRT } \\
\text { vs. RA2 }\end{array}$ & $\begin{array}{c}\mathrm{RA} 2, \\
\text { mean } \pm \mathrm{SD}\end{array}$ & $\begin{array}{c}\text { P for RA1 } \\
\text { vs. RA2 }\end{array}$ \\
\hline \multicolumn{7}{|l|}{ OARs (Gy) } \\
\hline Brainstem D2\% & $45.9 \pm 15.3$ & 0.96 & $45.5 \pm 16.0$ & 0.80 & $45.4 \pm 15.7$ & 0.80 \\
\hline $\mathrm{D} 50 \%$ & $20.2 \pm 16.8$ & 0.24 & $20.7 \pm 16.9$ & 0.88 & $19.7 \pm 16.1$ & $0.01^{\mathrm{c}}$ \\
\hline Lens RD2\% & $3.0 \pm 1.1$ & $0.01^{\mathrm{a}}$ & $2.6 \pm 0.99$ & $0.01^{\mathrm{b}}$ & $2.6 \pm 1.1$ & 0.88 \\
\hline $\mathrm{D} 50 \%$ & $2.3 \pm 1.0$ & $0.01^{\mathrm{a}}$ & $2.00 \pm 0.8$ & $0.01^{\mathrm{b}}$ & $2.0 \pm 0.9$ & 0.96 \\
\hline Lens LD2\% & $3.0 \pm 1.1$ & $0.04^{\mathrm{a}}$ & $2.6 \pm 0.9$ & 0.11 & $2.9 \pm 1.2$ & 0.29 \\
\hline D50\% & $2.4 \pm 0.9$ & 0.06 & $2.1 \pm 0.9$ & 0.14 & $2.2 \pm 0.9$ & 0.20 \\
\hline Optic nerve R D2\% & $6.8 \pm 5.1$ & 0.11 & $5.9 \pm 3.4$ & $0.01^{\mathrm{b}}$ & $5.9 \pm 3.9$ & 0.58 \\
\hline $\mathrm{D} 50 \%$ & $5.1 \pm 3.7$ & $0.01^{\mathrm{a}}$ & $4.3 \pm 3.0$ & $0.01^{\mathrm{b}}$ & $4.5 \pm 3.5$ & 0.20 \\
\hline Optic nerve L D2\% & $6.6 \pm 2.9$ & $0.01^{\mathrm{a}}$ & $6.2 \pm 2.6$ & 0.07 & $6.2 \pm 2.6$ & 0.72 \\
\hline $\mathrm{D} 50 \%$ & $4.8 \pm 2.4$ & 0.17 & $4.1 \pm 1.8$ & 0.58 & $4.4 \pm 1.9$ & 0.14 \\
\hline Optic chiasma D2\% & $21.4 \pm 14.5$ & 0.45 & $21.8 \pm 15.1$ & 0.96 & $21.1 \pm 14.9$ & $0.01^{\mathrm{c}}$ \\
\hline $\mathrm{D} 50 \%$ & $17.5 \pm 12.3$ & 0.88 & $16.8 \pm 12.7$ & 0.96 & $16.8 \pm 12.4$ & 0.80 \\
\hline Pituitary D2\% & $15.4 \pm 10.5$ & 0.24 & $14.3 \pm 9.4$ & 0.58 & $14.0 \pm 8.8$ & 0.45 \\
\hline D50\% & $13.0 \pm 9.0$ & 0.33 & $12.0 \pm 8.3$ & 0.20 & $11.8 \pm 7.8$ & 0.45 \\
\hline Hippocampus R D2\% & $53.89 \pm 8.20$ & 0.77 & $53.88 \pm 8.48$ & 0.99 & $53.88 \pm 8.49$ & 0.41 \\
\hline $\mathrm{D} 50 \%$ & $37.95 \pm 18.04$ & 0.19 & $39.49 \pm 17.36$ & 0.21 & $38.99 \pm 17.36$ & 0.33 \\
\hline Hippocampus L D2\% & $49.05 \pm 17.08$ & 0.64 & $48.59 \pm 18.33$ & 0.39 & $44.75 \pm 22.81$ & 0.41 \\
\hline $\mathrm{D} 50 \%$ & $38.82 \pm 20.62$ & 0.32 & $37.65 \pm 21.47$ & 0.22 & $37.72 \pm 20.88$ & 0.90 \\
\hline \multicolumn{7}{|l|}{ B-P (\%) } \\
\hline V5 & $84.6 \pm 18.1$ & 0.22 & $84.1 \pm 18.6$ & 0.08 & $84.0 \pm 18.3$ & 0.80 \\
\hline V10 & $75.8 \pm 17.5$ & 0.06 & $77.1 \pm 17.9$ & 0.11 & $77.2 \pm 17.6$ & 0.37 \\
\hline V15 & $62.8 \pm 14.6$ & 0.11 & $64.0 \pm 15.8$ & 0.06 & $65.4 \pm 14.1$ & 0.20 \\
\hline V20 & $53.1 \pm 12.6$ & 0.17 & $55.2 \pm 14.7$ & 0.17 & $53.8 \pm 13.1$ & 0.26 \\
\hline V25 & $44.3 \pm 12.4$ & 0.88 & $44.8 \pm 13.8$ & 0.07 & $43.3 \pm 12.6$ & 0.11 \\
\hline V30 & $36.1 \pm 11.5$ & 0.37 & $35.7 \pm 12.5$ & $0.01^{\mathrm{b}}$ & $34.3 \pm 11.4$ & 0.06 \\
\hline V35 & $27.6 \pm 9.8$ & 0.37 & $27.0 \pm 10.2$ & $0.03^{\mathrm{b}}$ & $25.8 \pm 9.5$ & 0.08 \\
\hline V40 & $20.1 \pm 7.9$ & 0.11 & $19.0 \pm 7.7$ & $0.03^{\mathrm{b}}$ & $18.3 \pm 7.3$ & 0.09 \\
\hline V45 & $13.9 \pm 6.3$ & $0.01^{\mathrm{a}}$ & $12.2 \pm 6.1$ & $0.01^{\mathrm{b}}$ & $13.0 \pm 6.0$ & 0.44 \\
\hline V50 & $8.4 \pm 5.6$ & $0.01^{\mathrm{a}}$ & $6.6 \pm 5.4$ & $0.01^{\mathrm{b}}$ & $6.6 \pm 5.5$ & 0.73 \\
\hline
\end{tabular}

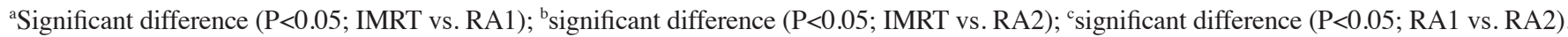
IMRT, intensity modulated radiotherapy; RA1, RapidArc with single arc; RA2, RapidArc with dual arc; SD, standard deviation; OAR, organs at risk; D2\%, near-maximum dose; D50\%, median dose; B-P, whole brain minus PTV2; Vn, related volume of the B-P receiving at least nGy.
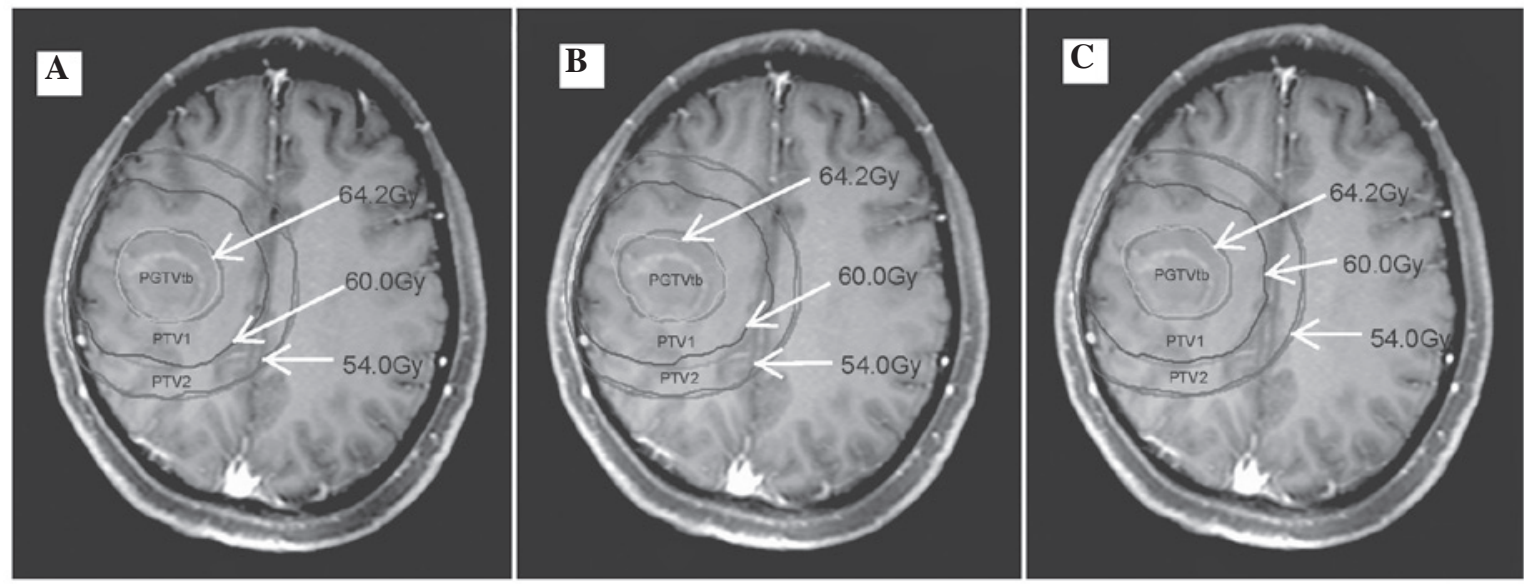

Figure 1. Dose distribution of a representative patient with axial views for (A) intensity modulated radiotherapy, and RapidArc with (B) a single arc and (C) a dual arc. 
A

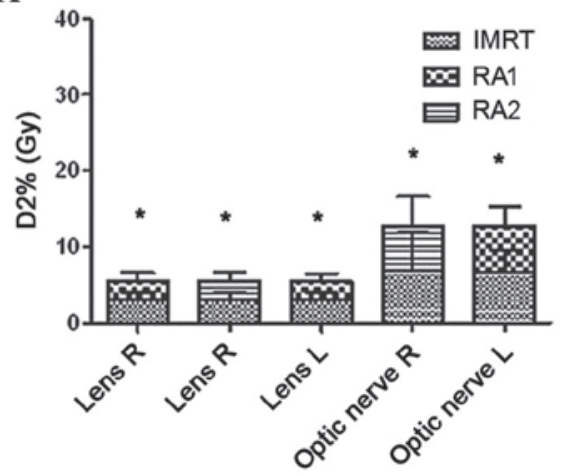

B

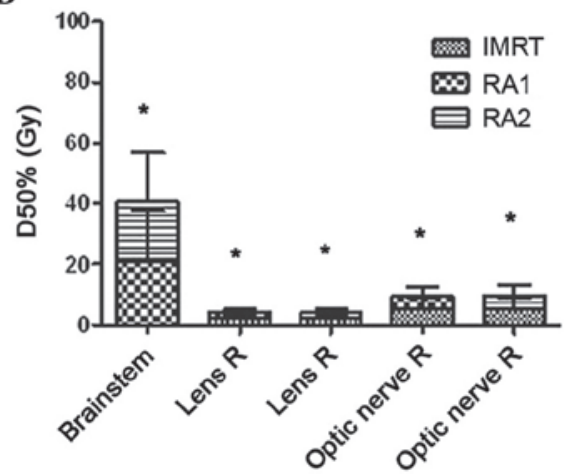

Figure 2. (A) D2\% and (B) D50\% of OARs. "P<0.05 with significant difference. D2\% of OARs: Lens R, RA1 vs. IMRT and RA2 vs. IMRT; Lens L, RA1 vs. IMRT; Optic nerve R, RA2 vs. IMRT; Optic nerve L, RA1 vs. IMRT. D50\% of OARs: Brainstem, RA1 vs. RA2; Lens R, RA1 vs. IMRT and RA2 vs. IMRT; Optic nerve R, RA1 vs. IMRT and RA2 vs. IMRT. OARs, organs at risk; L, left; R, right; IMRT, intensity modulated radiotherapy; RA1, RapidArc with single arc; RA2, RapidArc with dual arc; D2\%, near-maximum dose; D50\%, median dose.

A

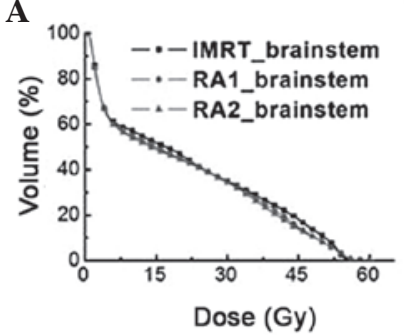

B
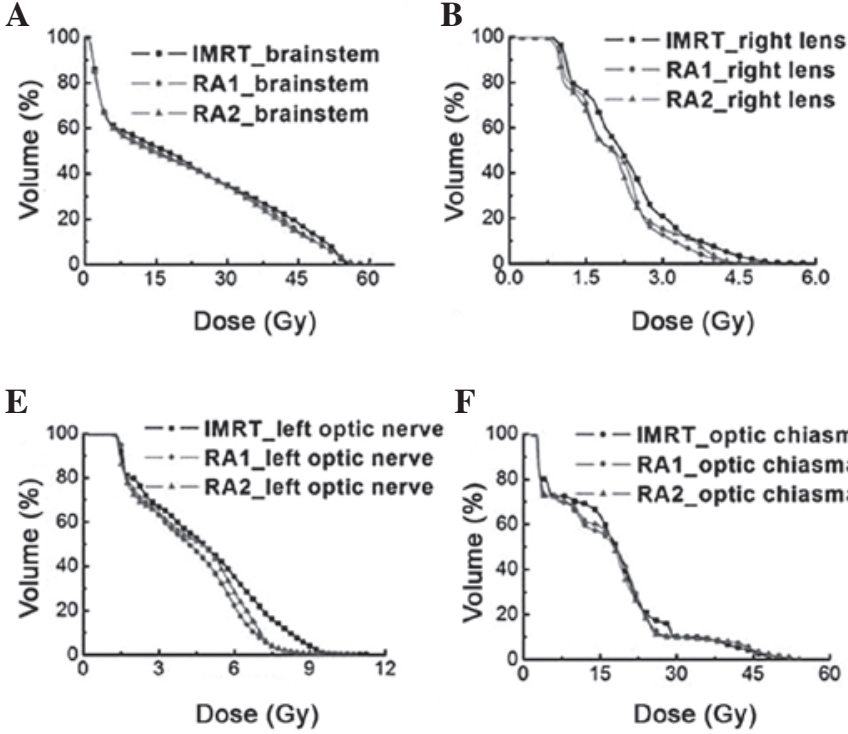

F

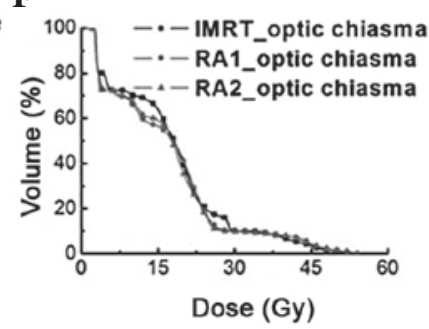

C

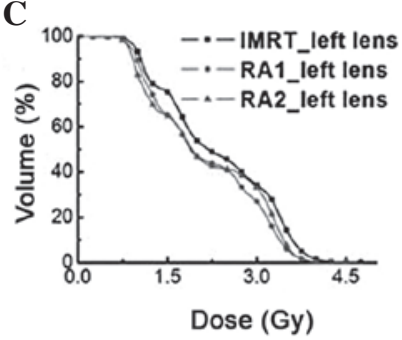

G

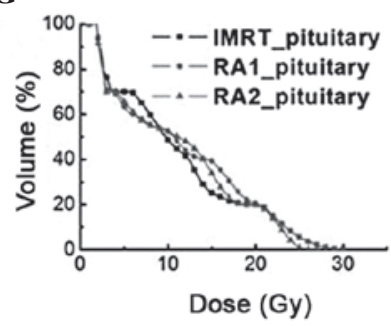

D

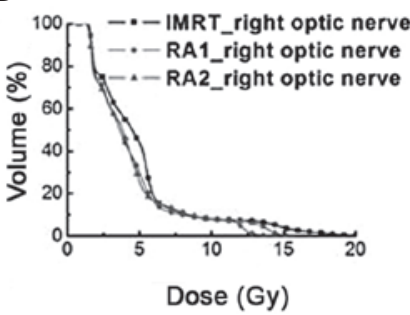

H

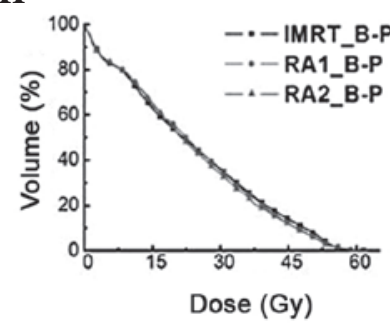

Figure 3. Mean dose-volume histogram of patients with IMRT, RA1, and RA2, showing results for the (A) brainstem, (B) right lens, (C) left lens, (D) right optic nerve, (E) left optic nerve, (F) optic chiasma, (G) pituitary and (H) B-P. IMRT, intensity modulated radiotherapy; RA1, RapidArc with single arc; RA2, RapidArc with dual arc; B-P, whole brain minus planned target volume 2.

malignant gliomas, and identified that PTV coverage was higher for IMRT (94.7\%) compared with that for RA1 $(90.5 \%)$ and 3D-CRT (81.2\%). The inhomogeneity was higher for 3D-CRT (8.2 Gy) compared with for RA1 (8.0 Gy), and lowest for IMRT $(6.8 \mathrm{~Gy})$. V5\% of healthy tissue, equivalent to a low-dose area, was lowest for 3D-CRT and highest for RA1. All OARs received a slightly lower dose by RA1 compared with IMRT or 3D-CRT. The number of MUs was 1.8 times lower for RA1 (321.1 \pm 58.8$)$ compared with IMRT (587.8 \pm 196.2$)$, and 1.4 times higher compared with 3D-CRT $(224.0 \pm 12.6)$. These results were similar to those in the present study in terms of coverage and homogeneity of PTV, however, the present study has shown that RA1 reduced the high dose volume in B-P, but compromised on sparing coverage and homogeneity of PTV. In contrast with the results of the present study, Munck Af Rosenschöld et al (17) reported an RA technique that tended to have a more conform target coverage compared with IMRT (not significant) in malignant gliomas. Panet-Raymond et al (4) demonstrated that significant differences were observed in CIs, with improved CIs noted in VMAT plans (IMRT, 0.88 and non-coplanar IMRT, 0.89 vs. VMAT, 0.917 and non-coplanar, VMAT 0.923; $\mathrm{P}<0.05$ ), whereas HIs were similar across the techniques evaluated (HI, 0.99 for all techniques) in fronto-temporal lobe high-grade glioma. It is hypothesized that the location of lesions and differences in the treatment plan strategies due to using co-planar or non-coplanar radiation techniques resulted in the different results of the dosimetric parameters in the above-mentioned studies.

The associations between the number of arcs with RapidArc and the optimal dose distribution and complexity of target volume have been studied previously (18). RapidArc plans have been extended to use more than one arc. In several cases, the use of two arcs rather than one has resulted in improved dose distributions (19). Verbakel et al (20) reported that, compared with IMRT, RA1 reduced target volume coverage and homogeneity, and RA2 improved the dosimetric distribution in target 
Table III. CI, HI, MU per fraction and treatment time for PGTVtb, PTV1, and PTV2 of IMRT, RA1, and RA2.

\begin{tabular}{lcccccc}
\hline Parameter & $\begin{array}{c}\text { IMRT } \\
\text { Mean } \pm \text { SD }\end{array}$ & $\begin{array}{c}\text { P for IMRT } \\
\text { vs. RA1 }\end{array}$ & $\begin{array}{c}\text { RA1 } \\
\text { Mean } \pm \text { SD }\end{array}$ & $\begin{array}{c}\text { P for IMRT } \\
\text { vs. RA2 }\end{array}$ & $\begin{array}{c}\text { RA2 } \\
\text { Mean } \pm \text { SD }\end{array}$ & $\begin{array}{c}\text { P for RA1 } \\
\text { vs. RA2 }\end{array}$ \\
\hline CI & & & & & & \\
PGTVtb & $0.79 \pm 0.04$ & $0.01^{\mathrm{a}}$ & $0.77 \pm 0.49$ & 0.96 & $0.80 \pm 0.05$ & $0.01^{\mathrm{c}}$ \\
PTV1 & $0.88 \pm 0.01$ & $0.01^{\mathrm{a}}$ & $0.85 \pm 0.02$ & 0.39 & $0.87 \pm 0.02$ & $0.01^{\mathrm{c}}$ \\
PTV2 & $0.87 \pm 0.02$ & $0.03^{\mathrm{a}}$ & $0.84 \pm 0.01$ & 0.05 & $0.88 \pm 0.02$ & $0.01^{\mathrm{c}}$ \\
HI & & & & & & \\
PGTVtb & $0.04 \pm 0.00$ & $0.03^{\mathrm{a}}$ & $0.05 \pm 0.01$ & 0.10 & $0.04 \pm 0.01$ & $0.02^{\mathrm{c}}$ \\
PTV1 & $0.11 \pm 0.00$ & $0.01^{\mathrm{a}}$ & $0.12 \pm 0.01$ & 0.17 & $0.11 \pm 0.01$ & $0.02^{\mathrm{c}}$ \\
PTV2 & $0.23 \pm 0.01$ & $0.01^{\mathrm{a}}$ & $0.24 \pm 0.02$ & 0.09 & $0.23 \pm 0.01$ & $0.01^{\mathrm{c}}$ \\
MU per fraction & $630.30 \pm 98.68$ & $0.01^{\mathrm{a}}$ & $363.30 \pm 40.97$ & $0.01^{\mathrm{b}}$ & $356.60 \pm 37.30$ & 0.45 \\
Treatment time & $302.00 \pm 25.30$ & $<0.01^{\mathrm{a}}$ & $73.10 \pm 7.71$ & $<0.01^{\mathrm{b}}$ & $186.50 \pm 15.83$ & $<0.01^{\mathrm{c}}$ \\
\hline
\end{tabular}

Significant difference of ${ }^{\mathrm{a} I M R T}$ vs. RA1, ${ }^{\mathrm{b}} \mathrm{IMRT}$ vs. RA2 and ${ }^{\mathrm{c}} \mathrm{RA} 1 \mathrm{vs.}$ RA2. IMRT, intensity modulated radiotherapy; RA1, RapidArc with single arc; RA2, RapidArc with dual arc; SD, standard deviation; CI, conformal index; HI, homogeneity index; MU, monitor unit.

volume with lower doses to OARs. Similar results were made by Vanetti et al (21), who concluded that RA1 and RA2 exhibited certain improvements in sparing OARs and healthy tissue. Target coverage and homogeneity results improved with RA2 plans compared with those of RA1 and IMRT in head-and-neck cancer patients. Clivio et al (22) analyzed 10 patients with anal canal cancer who were treated with RA1, RA2 or IMRT. All techniques resulted in similar target coverage, and in terms of sparing OARs, RA2 was superior to RA1 and IMRT. The present study has shown that RA1 was inferior to RA2 in terms of coverage of PTV and in sparing OARs, and that normal brain tissue received low-dose irradiation of malignant gliomas involving the parietal lobe. The results reported for previous studies were similar to those obtained in the present study.

A body of amassed evidence has indicated that radiation can induce cancer in the human. Radiation-induced neoplasms following fractionated radiation therapy in the CNS have been well documented, and it is considered that the risk of developing a radiation induced tumor is $\sim 1-3 \%$ (23-25). Three cases of radiation-induced neoplasms have been reported following radiosurgery (26-28). The risk of a radiation-associated brain tumor in survivors of childhood cancer is positively associated with a young age at time of radiation ( $<6$ years), higher radiation doses ( $>30 \mathrm{~Gy}$ ), and concomitant treatment with antimetabolites (particularly in patients with thiopurine methyltransferase deficiency) (29-31). Information regarding radiation dose-response associations and subsequent tumors of the CNS is sparse. Neglia et al (29) identified statistically significant radiation dose-response associations for gliomas and meningiomas in childhood cancer survivors, and the relative risks at a specified dose were higher for meningiomas than for gliomas. IMRT has the potential to increase the number of radiation-induced second cancers $(32,33)$. There are two reasons why the IMRT may result in an increase in second malignancies compared with conventional radiotherapy. First, the change from IMRT involves the use of more fields, and, as a consequence, a bigger volume of normal tissue is exposed to lower doses. Secondly, delivery of a specified dose to the isocenter from a modulated field, deliv- ered by IMRT, will require the accelerator to be energized for longer (thus more monitor units are required) compared with delivering the identical dose from an unmodulated field (34). There are estimates in the literature that the number of MUs in an IMRT plan is two to three times higher compared with a conventional radiotherapy plan, with an increase in the incidence of radiation-induced secondary malignancies from $1-1.75 \%$ for patients who survive for 10 years or more $(34,35)$. The present study has demonstrated that RA1 and RA2 markedly reduced the MUs per fraction, and the median and high dose volume of the healthy brain compared with those in IMRT; therefore, RA1 and RA2 are likely to decrease the incidence of radiation-induced second cancer in the healthy brain.

Late sequelae of radiotherapy, which appear from 6 months to a number of years following treatment, are usually irreversible and progressive. They are considered to be due to white matter damage from vascular injury, demyelination and necrosis. The pathophysiology of radiation-induced neurocognitive damage is complex, and involves intercellular and intracellular interactions between vasculature and parenchymal cells, particularly oligodendrocytes, which are important for myelination (36). Corn et al (37) performed a phase I/II randomized trial to analyze the association between white matter changes and serial imaging scans (i.e. MRI and CT scans) that are associated with bis-chlorethyl nitrosourea and hyper-fractionated cranial irradiation. They observed grade 3 or worse changes in 8.3, 20.0 and $36.5 \%$ of patients in the low-, intermediate- and high-dose groups, respectively. For a toxicity of grade 3 or worse, a chi-squared test revealed P-values of 0.04 (low vs. intermediate dose), 0.09 (intermediate vs. high dose), and 0.0005 (low vs. high dose). The present study indicated that V45-V50 in RA1, and V35-V50 in RA2, of B-P were significantly less compared with those in IMRT; therefore, RA1 and RA2 may be decrease white matter damage and lessen the sequelae of brain irradiation.

Radiation damage to cells is not always lethal. It is well documented that sublethal damage caused by radiation may be repaired within hours following irradiation. Sublethal damage 
repair occurs not only in normal tissues, but also in tumors, and takes place not only between fractions, but also during irradiation. Therefore, the treatment time of each fraction affects the level of cell survival. As the treatment time is extended, the biological effect of a specified dose is generally reduced. The effect of prolonged delivery times of IMRT treatments on tumor control has been studied by Wang et al (38). When the identical prescribed doses are delivered with more MUs in IMRT, the clinical results may be worse when compared with the outcomes in RapidArc with fewer MUs. Long treatment time resulted in a reduction of local control rate. On the other hand, prolonged beam delivery time of IMRT compared with RapidArc may worsen the accuracy of treatment, due to increased intrafractional patient motion; in addition, patient throughput is reduced, with economical consequences. The present study has shown that RA1 and RA2 significantly decreased MUs per fraction and the treatment time compared with IMRT in gliomas involving the parietal lobe, and the treatment time of radiotherapy was subsequently reduced, which led to a decrease in sublethal damage repair.

Although statistically significant differences were observed in the dosimetric parameters of specific OARs among IMRT and the RA1 and RA2 plans, the difference between the dosimetric parameters is small, and so it is not clear whether RA1 and RA2 are able to reduce radiation-induced cancer and late sequelae of radiotherapy, including brain radionecrosis and cognition impairment. Teoh et al (39) considered that the distinction of dosage parameters of OARs and normal tissue between VMAT and fixed-field IMRT is less clear. The data suggest that, for most tumor sites, VMAT and fixed-field IMRT do produce largely equivalent target volume coverage, dose conformity and homogeneity. The absolute difference in dosimetric parameters reported as being statistically significant in certain of the planning studies is comparatively small, and may not be clinically significant. In the future, a prospective study will be undertaken to clarify the effect of RA1 and RA2 on the rate of radiation-induced cancer and late sequelae of radiotherapy compared with those of IMRT. The subsequent selection of RapidArc will depend on its availability, the size, location and morphology of the brain tumor, and economic conditions.

\section{Acknowledgements}

The present study was supported by the Hunan Province Development and Reform Committee Science Research Fund (nos. 2010-1060 and 2014-463), the Hunan Province Science and Technology Program (no. 2011SK3223), the Hunan Province Science and Technology Program (no. 2011FJ4184), the Hunan Provincial Natural Science Foundation of China (no. 2012JJ5043), and the Neuro-oncology research project, Chinese Society of Neuro-oncology (no. CNSO-2014-MSD14).

\section{References}

1. MacDonald SM, Ahmad S, Kachris S, Vogds BJ, DeRouen M, Gittleman AE, DeWyngaert K and Vlachaki MT: Intensity modulated radiation therapy versus three-dimensional conformal radiation therapy for the treatment of high grade glioma: A dosimetric comparison. J Appl Clin Med Phys 8: 47-60, 2007.
2. Lorentini S, Amelio D, Giri MG, Fellin F, Meliado G, Rizzotti A, Amichetti M and Schwarz M: IMRT or 3D-CRT in glioblastoma? A dosimetric criterion for patient selection. Technol Cancer Res Treat 12: 411-420, 2013

3. Shaffer R, Nichol AM, Vollans E, Fong M, Nakano S, Moiseenko V, Schmuland M, Ma R, McKenzie M and Otto K: A comparison of volumetric modulated arc therapy and conventional intensity-modulated radiotherapy for frontal and temporal high-grade gliomas. Int J Radiat Oncol Biol Phys 76: 1177-1184, 2010.

4. Panet-Raymond V, Ansbacher W, Zavgorodni S, Bendorffe B, Nichol A, Truong PT, Beckham W and Vlachaki M: Coplanar versus noncoplanar intensity-modulated radiation therapy (IMRT) and volumetric-modulated arc therapy (VMAT) treatment planning for fronto-temporal high-grade glioma. J Appl Clin Med Phys 13: 3826, 2012.

5. Louis DN, Ohgaki H, Wiestler OD, Cavenee WK, Burger PC, Jouvet A, Scheithauer BW and Kleihues P: The 2007 WHO classification of tumours of the central nervous system. Acta Neuropathol. 114: 97-109, 2007.

6. Stupp R, Mason WP, Van Den Bent MJ, Weller M, Fisher B, Taphoorn MJ, Belanger K, Brandes AA, Marosi C, Bogdahn U, et al: Radiotherapy plus concomitant and adjuvant temozolomide for glioblastoma. N Engl J Med 352: 987-996, 2005.

7. Ali AN, Ogunleye T, Hardy CW, Shu HK, Curran WJ and Crocker IR: Improved hippocampal dose with reduced margin radiotherapy for glioblastoma multiforme. Radiat Oncol. 9: 20, 2014.

8. Chui CS, LoSasso T and Spirou S: Dose calculation for photon beams with intensity modulation generated by dynamic jaw or multileaf collimations. Med Phys 21: 1237-1244, 1994.

9. Spirou SV and Chui C-S: A gradient inverse planning algorithm with dose-volume constraints. Med Phys 25: 321-333, 1998.

10. Bragg CM, Wingate $\mathrm{K}$ and Conway J: Clinical implications of the anisotropic analytical algorithm for IMRT treatment planning and verification. Radiother Oncol 86: 276-284, 2008.

11. Knöös T, Wieslander E, Cozzi L, Brink C, Fogliata A, Albers D, Nyström $\mathrm{H}$ and Lassen S: Comparison of dose calculation algorithms for treatment planning in external photon beam therapy for clinical situations. Phys Med Biol 51: 5785-5807, 2006.

12. Ulmer W, Pyyry J and Kaissl W: A 3D photon superposition/convolution algorithm and its foundation on results of Monte Carlo calculations. Phys Med Biol 50: 1767-1790, 2005.

13. Cozzi L, Dinshaw KA, Shrivastava SK, Mahantshetty U, Engineer R, Deshpande DD, Jamema SV, Vanetti E, Clivio A, Nicolini G and Fogliata A: A treatment planning study comparing volumetric arc modulation with RapidArc and fixed field IMRT for cervix uteri radiotherapy. Radiother Oncol 89: 180-191, 2008.

14. van't Riet A, Mak AC, Moerland MA, Elders LH and van der Zee W: A conformation number to quantify the degree of conformality in brachytherapy and external beam irradiation: Application to the prostate. Int J Radiat Oncol Biol Phys 37: 731-736, 1997.

15. Wu Q, Mohan R, Morris M, Lauve A and Schmidt-Ullrich R: Simultaneous integrated boost intensity-modulated radiotherapy for locally advanced head-and-neck squamous cell carcinomas. I: Dosimetric results. Int J Radiat Oncol Biol Phys 56: 573-585, 2003.

16. Wagner D, Christiansen H, Wolff H and Vorwerk H: Radiotherapy of malignant gliomas: Comparison of volumetric single arc technique (RapidArc), dynamic intensity-modulated technique and 3D conformal technique. Radiother Oncol 93: 593-596, 2009.

17. Munck Af Rosenschöld P, Engelholm S, Ohlhues L, Law I, Vogelius I and Engelholm SA: Photon and proton therapy planning comparison for malignant glioma based on $\mathrm{CT}$, FDG-PET, DTI-MRI and fiber tracking. Acta Oncol 50: 777-783, 2011.

18. Guckenberger M, Richter A, Krieger T, Wilbert J, Baier K and Flentje M: Is a single arc sufficient in volumetric-modulated arc therapy (VMAT) for complex-shaped target volumes? Radiother Oncol 93: 259-265, 2009.

19. Palma DA, Verbakel WF, Otto K and Senan S: New developments in arc radiation therapy: A review. Cancer Treat Rev 36: 393-399, 2010.

20. Verbakel WF, Senan S, Cuijpers JP, Slotman BJ and Lagerwaard FJ: Rapid delivery of stereotactic radiotherapy for peripheral lung tumors using volumetric intensity-modulated arcs. Radiother Oncol 93: 122-124, 2009. 
21. Vanetti E, Clivio A, Nicolini G, Fogliata A, Ghosh-Laskar S, Agarwal JP, Upreti RR, Budrukkar A, Murthy V, Deshpande DD, et al: Volumetric modulated arc radiotherapy for carcinomas of the oro-pharynx, hypo-pharynx and larynx: A treatment planning comparison with fixed field IMRT. Radiother Oncol 92: 111-117, 2009.

22. Clivio A, Fogliata A, Franzetti-Pellanda A, Nicolini G, Vanetti E, Wyttenbach $\mathrm{R}$ and Cozzi L: Volumetric-modulated arc radiotherapy for carcinomas of the anal canal: A treatment planning comparison with fixed field IMRT. Radiother Oncol 92: 118-124, 2009.

23. Ron E, Modan B, Boice JD Jr, Alfandary E, Stovall M, Chetrit A and Katz L: Tumors of the brain and nervous system after radiotherapy in childhood. N Engl J Med 319: 1033-1039, 1988.

24. Simmons NE and Laws ER Jr: Glioma occurrence after sellar irradiation: Case report and review. Neurosurgery 42: 172-178, 1998.

25. Tsang RW, Laperriere NJ, Simpson WJ, Brierley J, Panzarella T and Smyth HS: Glioma arising after radiation therapy for pituitary adenoma. A report of four patients and estimation of risk. Cancer 72: 2227-2233, 1993.

26. Kaido T, Hoshida T, Uranishi R, Akita N, Kotani A, Nishi N and Sakaki T: Radiosurgery-induced brain tumor. Case report. J Neurosurg 95: 710-713, 2001.

27. Shamisa A, Bance M, Nag S, Tator C, Wong S, Norén G and Guha A: Glioblastoma multiforme occurring in a patient treated with gamma knife surgery. Case report and review of the literature. J Neurosurg 94: 816-821, 2001.

28. Yu JS, Yong WH, Wilson D and Black KL: Glioblastoma induction after radiosurgery for meningioma. Lancet 356 1576-1577, 2000.

29. Neglia JP, Robison LL, Stovall M, Liu Y, Packer RJ, Hammond S, Yasui Y, Kasper CE, Mertens AC, Donaldson SS, et al: New primary neoplasms of the central nervous system in survivors of childhood cancer: A report from the childhood cancer survivor study. J Natl Cancer Inst 98: 1528-1537, 2006.

30. Relling MV, Rubnitz JE, Rivera GK, Boyett JM, Hancock ML, Felix CA, Kun LE, Walter AW, Evans WE and Pui CH: High incidence of secondary brain tumours after radiotherapy and antimetabolites. Lancet 354: 34-39, 1999.
31. Walter AW, Hancock ML, Pui CH, Hudson MM, Ochs JS, Rivera GK, Pratt CB, Boyett JM and Kun LE: Secondary brain tumors in children treated for acute lymphoblastic leukemia at $\mathrm{St}$ Jude Children's Research Hospital. J Clin Oncol 16: 3761-3767, 1998.

32. Followill D, Geis P and Boyer A: Estimates of whole-body dose equivalent produced by beam intensity modulated conformal therapy. Int J Radiat Oncol Biol Phys 38: 667-672, 1997.

33. Kry SF, Salehpour M, Followill DS, Stovall M, Kuban DA, White RA and Rosen II: The calculated risk of fatal secondary malignancies from intensity-modulated radiation therapy. Int J Radiat Oncol Biol Phys 62: 1195-1203, 2005.

34. Hall EJ and Wuu CS: Radiation-induced second cancers: The impact of 3D-CRT and IMRT. Int J Radiat Oncol Biol Phys 56: 83-88, 2003.

35. Gershkevitsh E, Clark CH, Staffurth J, Dearnaley DP and Trott KR: Dose to bone marrow using IMRT techniques in prostate cancer patients. Strahlenther Onkol 181: 172-178, 2005.

36. Halperin EC, Perez CA and Brady LW: Principles and Practice of Radiation Oncology, 5th Edn., 730, Lippincott Williams \& Wilkins, 2008

37. Corn BW, Yousem DM, Scott CB, Rotman M, Asbell SO, Nelson DF, Martin L and Curran WJ Jr: White matter changes are correlated significantly with radiation dose. Observations from a randomized dose-escalation trial for malignant glioma (Radiation therapy oncology group 83-02). Cancer 74: 2828-2835, 1994.

38. Wang JZ, Li XA, D'Souza WD and Stewart RD: Impact of prolonged fraction delivery times on tumor control: A note of caution for intensity-modulated radiation therapy (IMRT). Int J Radiat Oncol Biol Phys 57: 543-552, 2003.

39. Teoh M, Clark CH, Wood K, Whitaker S and Nisbet A: Volumetric modulated arc therapy: A review of current literature and clinical use in practice. Br J Radiol 84: 967-996, 2011. 